\title{
Mind-Body Medicine for Multiple Sclerosis: A Systematic Review
}

\author{
Angela Senders, ${ }^{1}$ Helané Wahbeh, ${ }^{1}$ Rebecca Spain, ${ }^{1,2}$ and Lynne Shinto ${ }^{1}$ \\ ${ }^{1}$ Department of Neurology, Oregon Health \& Science University, Portland, OR 97239, USA \\ ${ }^{2}$ Department of Neurology, Portland Veterans Affairs Medical Center, Portland, OR 97239, USA
}

Correspondence should be addressed to Angela Senders, senders@ohsu.edu

Received 8 August 2012; Accepted 6 October 2012

Academic Editor: D. N. Bourdette

Copyright ( $) 2012$ Angela Senders et al. This is an open access article distributed under the Creative Commons Attribution License, which permits unrestricted use, distribution, and reproduction in any medium, provided the original work is properly cited.

Background. Mind-body therapies are used to manage physical and psychological symptoms in many chronic health conditions. Objective. To assess the published evidence for using mind-body techniques for symptom management of multiple sclerosis. Methods. MEDLINE, PsycINFO, and Cochrane Clinical Trials Register were searched from inception to March 24, 2012. Eleven mind-body studies were reviewed (meditation, yoga, biofeedback, hypnosis, relaxation, and imagery). Results. Four high quality trials (yoga, mindfulness, relaxation, and biofeedback) were found helpful for a variety of MS symptoms. Conclusions. The evidence for mind-body medicine in MS is limited, yet mind-body therapies are relatively safe and may provide a nonpharmacological benefit for MS symptoms.

\section{Introduction}

Multiple sclerosis (MS) is a chronic neurological disorder with emotional, cognitive, and physical consequences. Patients can experience a diverse array of symptoms including impaired mobility, sensory disturbance, chronic pain, fatigue, bladder and bowel dysfunction, depression, and cognitive impairment. Patients report high levels of stress, independent of physical disability [1-3], and the risk of developing stress-related disorders like anxiety and depression is high ( $25 \%$ and $34-50 \%$ lifetime prevalence, resp.) [47]. There is no cure for MS, and both disease-modifying and symptomatic therapies have limitations in compliance due to side effects and cost $[8,9]$.

Mind-body therapies are used by a growing number of American adults and children [10]. In a survey of 1,110 MS patients, $32 \%$ of respondents had used mindbody modalities and reported a high perceived benefit [11]. The National Center for Complementary and Alternative Medicine defines mind-body therapies as those that integrate the brain, mind, body, and behavior, with the intent to use the mind to affect physical functioning and promote health [12]. Examples of such modalities include meditation, yoga, relaxation techniques, breathwork, visual imagery, hypnotherapy, and biofeedback. While these practices vary in technique and application, they share a common objective to enhance the capacity of the mind to improve physical and psychological wellbeing.

Many mind-body therapies can be taught in a clinical setting and then subsequently used at home by the patient in a self-directed way, making them ideal tools to promote increased self-efficacy. Mind-body techniques often have a calming effect on the autonomic nervous system [13] and thus may be helpful for conditions where psychological stress is a factor. From this perspective, mind-body interventions hold strong potential for use in MS. Broad reviews have addressed the use of mind-body therapies in general practice [14] and neurology [15], yet no comprehensive review of evidence for MS exists. The objective of this paper is to systematically review the published evidence for mind-body therapies for symptom management in MS.

\section{Methods}

One reviewer performed the searches, assessed inclusion criteria, and extracted data on trial design and outcomes. Two reviewers independently assessed methodology and risk 
of bias for each study included in this paper. Because we expected the sample size of mind-body studies to be small, we intentionally kept inclusion criteria broad.

2.1. Types of Studies. We included only controlled trials in this paper.

2.2. Types of Participants. We included studies of any kind of MS. Studies that did not verify diagnosis of MS or use accepted diagnostic criteria for their time were included in this paper; however, their risk of bias is included in our analysis. We added the category "MS diagnosis confirmation and criteria" to the Cochrane Risk of Bias Table for this purpose.

2.3. Types of Interventions. Mind-body therapies included meditation and mindfulness, yoga, hypnosis, biofeedback, relaxation, imagery, tai chi, and qi gong.

2.4. Types of Outcome Measures. Included were studies with any MS outcome related to physical or psychosocial wellbeing. Specific outcomes were not defined.

2.5. Search Strategy for Identification of Studies. One reviewer (A. Senders) searched the electronic databases MEDLINE, PsychInfo, and Cochrane Clinical Trials Register. Databases were searched in Ovid (http://www.ovid.com/) from inception to March 24, 2012, using the exploded medical subject headings (MeSH): Mind-Body Therapies; Mind-Body Relations, Metaphysical; Therapeutics; Hypnosis; Yoga; Imagery; Biofeedback; Tai Ji; Meditation; Relaxation; Muscle Relaxation; Relaxation Therapy; Breathwork; Breathing Exercises. Tai chi, Qi Gong, Breathing Technique, and Mindfulness were searched as keywords. These MeSH terms and keywords were then combined with MS terms Multiple Sclerosis; Multiple Sclerosis, Chronic Progressive; Multiple Sclerosis, Relapsing-Remitting. A medical librarian was consulted to review the search protocol. Only studies reported in the English language were considered in this review. A manual review of references or related reviews was not completed. The specific search strategy can be found in the Appendix.

2.6. Selection of Studies. One author (A. Senders) conducted the initial search. Potential publications were selected by screening titles and abstracts for clinical trials that used a mind-body intervention for any type of multiple sclerosis. Final selections were made by screening full articles for the following inclusion criteria: any type of MS, any mindbody intervention, any kind of comparator group, and any outcome related to physical or mental wellbeing.

2.7. Data Extraction and Management. Data extraction from each study was performed by one author (A. Senders) using a data collection form that included study design, participant characteristics, type and duration of intervention, comparator treatment, primary and secondary outcome measures, whether or not homework was prescribed, adherence to homework, dropout rate/loss to follow up, and summary of results. Authors of reviewed studies were not contacted for missing information or clarification.

2.8. Assessment of Risk of Bias in Included Studies. The methodological quality of clinical trials was assessed according to the Cochrane Risk of Bias Tool [36]. Assessments of bias were independently performed for each paper in duplicate (AS, HW, or LS) and any disagreements were recorded and resolved through discussion.

2.9. Issues of Heterogeneity. One objective of this paper was to grade the body of evidence for mind-body medicine in MS using the Grading of Recommendations Assessment, Development and Evaluation tool (GRADE) [37]. However, a meta-analysis or reliable grading of the body of evidence is not feasible due to a large amount of clinical and methodological variability between studies. Instead we describe and report on the state of the published evidence to date.

\section{Results}

Mind-body therapies are briefly described in Table 1. While the therapies are described in discrete categories, considerable crossover exists between them. For example, guided imagery is often included as a part of meditation and relaxation protocols, yoga has a meditative component to it, and biofeedback for stress reduction often involves relaxation or autogenic training.

3.1. Search Results. Searching three electronic databases revealed a total of 106 articles. Titles and available abstracts were screened for clinical trials that used a mind-body intervention for MS; 87 articles were excluded because they were nonintervention papers and 19 articles were identified for further review (see Figure 1). From this, 10 studies were selected based on the inclusion criteria above. Six mindbody modalities are included in this paper: two studies of hypnosis, one of yoga, two of relaxation, two of mindfulness, two of biofeedback, and one study of guided imagery. Of the two mindfulness studies, one incorporated the principle of "mindfulness of movement" from tai chi/qi gong. We categorized this study as a mindfulness trial. No specific studies of tai chi or qi gong were identified.

3.2. Description of Included Studies. A summary of the characteristics of the 10 studies is provided in Table 2. There were seven randomized controlled trials (RCTs) $[25,27,30-34]$, one controlled trial in which the first eight eligible subjects were enrolled in the experimental group and the subsequent 14 subjects were randomized [28], one prospective cohort study with matched controls [26], and one prospective repeated measures design that compared within subject change between four different hypnosis interventions [29]. Subjects were recruited from local MS Society chapters, outpatient neurology clinics, community advertisements, rehabilitation centers, and local practitioners. Sample sizes varied from 20 to 150; mean 
TABLE 1: Description of mind-body therapies and percent use by the general public.

\begin{tabular}{l} 
Modality $\quad \begin{array}{c}\text { Use by the } \\
\text { general public } \\
\text { (\%) [10] }\end{array}$ \\
\hline (i) A mental training that is a state of being more than a task. Practices incorporate self-observation and \\
awareness, emotional and attentional regulatory strategies, and the cultivation of an attitude of acceptance. \\
(ii) Many forms exist, share some distinctive features but vary in purpose and technique. \\
(iii) Most widely researched forms include transcendental meditation and mindfulness meditation. \\
(a) Transcendental meditation: a silent word or phrase (a mantra) is repeated in order to reduce mental \\
activity. \\
(b) Mindfulness meditation: practitioners cultivate an open, nonjudgmental awareness of both internal \\
experiences (thoughts, emotions, and bodily sensations) and external experiences (sights, sounds) in \\
the present moment. Has been formalized for clinical intervention with mindfulness-based stress \\
reduction, a program that is an amalgam of several mind-body techniques, including mindfulness \\
meditation, breathing exercises, yoga postures, and relaxation techniques.
\end{tabular}

(i) Incorporates physical postures, breathing, meditation into a multifaceted approach to physical/mental wellbeing.

(ii) Many different practices of yoga, each varying in focus.

(a) Hatha yoga is typically gentle with an emphasis on poses and breathwork.

(b) Ashtanga and Vinyasa yoga are more physically demanding, moving from posture to posture without stopping.

Yoga (c) Iyengar yoga is most concerned with precision of poses, encourages prop use to attain correct alignment.

(d) Bikram Yoga is practiced in a heated room (typically $105^{\circ} \mathrm{F}$ ).

(e) Kundalini yoga incorporates an added emphasis on the breath in conjunction with physical poses.

(iii) Like meditation, the practice of yoga cultivates a way of being rather than performing a task, although, Western practices that focus on exercise and physical health rather than awareness, insight, or spirituality have emerged.

(i) Relaxed state of focused, inward attention in which peripheral awareness is reduced.

(ii) Attaining alteration of consciousness involves absorption, dissociation, and suggestibility.

(a) Absorption: deep immersion in an internal experience.

(b) Dissociation: disconnect from peripheral events that would normally be conscious (perceptions, thoughts, emotions, or sensory activity).

Hypnosis $\quad$ (c) Suggestibility: suspension of conscious editing (not asking "why?"), respond to suggestion more readily.

(iii) Therapist may use guided imagery or hypnotic suggestion to help the person to understand behavioral patterns and envision making desired change.

(iv) Self-hypnosis techniques can be used at home, reducing the cost of therapy and encouraging self-efficacy.

(i) Electrodes placed on the body provide feedback to the patient about peripheral physiological markers like heart rate, breathing rate, muscle tension, or electrodermal activity.

(ii) Neurofeedback uses scalp electrodes to measure EEG activity.

(iii) Information relayed with visual or auditory cues; patient can attempt to change thoughts, emotions, or

Biofeedback

behaviors in order to control physiological reactions, such as slowing the heart rate or relaxing certain muscles.

(iv) Strategies are developed and refined with a practitioner, then utilized in real time outside the therapeutic encounter.

(v) Portable biofeedback devices available to enhance relaxation.

(vi) Individualized treatment goals depend on the specific condition being addressed.

(i) Reduces reactivity to physical, psychosocial, and environmental stressors by reducing sympathetic nervous system arousal and enhancing parasympathetic response [16].

(ii) The physiological counterpart of the fight-or-flight, response [17].

(iii) Jacobson's progressive muscular relaxation technique and autogenic training are formalized relaxation

Relaxation techniques, all the mind-body therapies initiate some kind of relaxation response.
(iv) Often incorporates breathing techniques that create awareness of breathing rate, rhythm, and volume.

(v) Voluntary control of breathing patterns influences autonomic nervous system functions: heart rate variability, cardiac vagal tone, and CNS excitation as indicated by EEG and MRI [18].

(vi) Relaxation training enhances awareness of nervous system activation; patients can employ techniques at any time to reduce reactivity. 
Table 1: Continued.

\begin{tabular}{|c|c|c|}
\hline Modality & Description & $\begin{array}{c}\text { Use by the } \\
\text { general public } \\
(\%)[10]\end{array}$ \\
\hline
\end{tabular}

(i) Most prominent forms are guided imagery and motor imagery.

(a) Guided imagery: involves visualization and imagination, goal of evoking a state of relaxation or a specific outcome (visualizing the repair of myelin, or one's white blood cells attacking a tumor). (b) Motor imagery: patient relives the sensations of undertaking a skilled movement without actually doing the movement [19]. Physiologic similarities between physically executed and imagined

Imagery movements have been noted in motor evoked potentials, MRI, PET, and EMG studies [20-24].

(ii) Can engage visual, tactile, kinesthetic, olfactory, gustatory, or any combination of these senses.

(iii) Imagery typically developed and refined with a therapist, then practiced regularly outside the

therapeutic encounter. Motor imagery is used in addition to physical therapy or exercise and not as an isolated treatment.

(iv) Tailored to condition and abilities, it should be practiced in patient's own context to be meaningful for their progress.

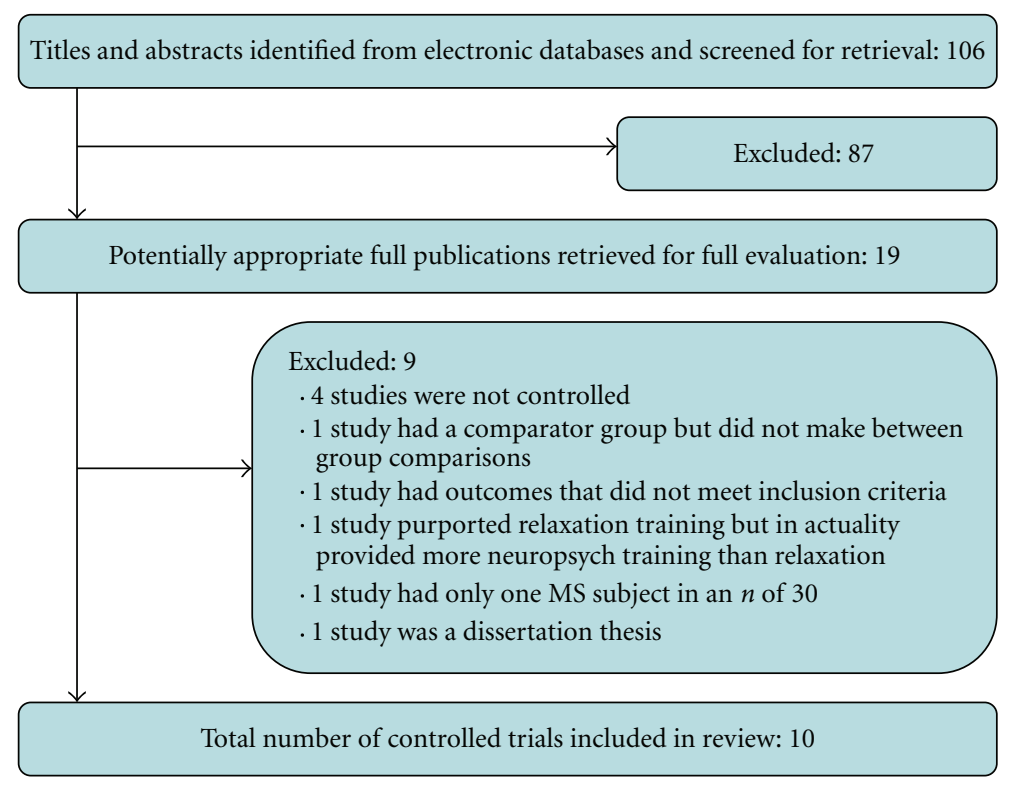

Figure 1: Flow diagram of literature search and study selection process.

sample size was $46.6 \pm 40.1$. In total, 466 people with MS were studied. Only three of the studies reported MS type [25, 26, 30]. Two studies used McDonald criteria [38] to confirm diagnosis $[25,27]$, three reported diagnosis confirmation by a neurologist but did not discuss criteria $[26,31,32]$, two studies reported confirmed diagnoses but did not report by who or how $[30,33]$, and three studies did not report whether MS diagnosis was confirmed or not $[28,29,34]$. Five studies measured participants' baseline Expanded Disability Status Scale (EDSS) scores [25, 27, 3032]; three of these studies provided mean EDSS scores for intervention and comparator groups. The EDSS is a measure of neurological impairment from 0 (normal neurological exam) to 10.0 (dead). Patients with scores in the 1.0-3.5 range have mild disability with no limitation of walking, 4.0-6.5 have increasing difficulty walking, and those 7.0 or higher require a wheelchair [39]. The average baseline EDSS for these five studies ranged from 2.9 to 5.9. The duration of interventions varied from four weeks (one training session a week) to 6 months (one training session a week). Assessment tools varied greatly and included a range of subjective and objective measures consisting of physical (disability, pain intensity, fatigue, incontinence, and symptom checklists), psychological (mood profiles, anxiety, pain catastrophizing, and sense of control over health), cognitive (attention, memory, and executive function), and quality of life measures. Eight studies specifically acknowledged asking participants to practice techniques on their own as homework [25, 27$30,32-34]$, and six of these studies made some attempt to quantify homework adherence $[25,27,28,30,32,33]$. Dropout rates and loss to follow-up ranged from $0 \%$ to $33 \%$.

3.3. Methodological Quality of Included Studies. Table 3 summarizes the risk of bias for each study. Review authors' judgments are categorized as High risk of bias (-), Unclear risk of bias (?), or Low risk of bias (+). Assessments 


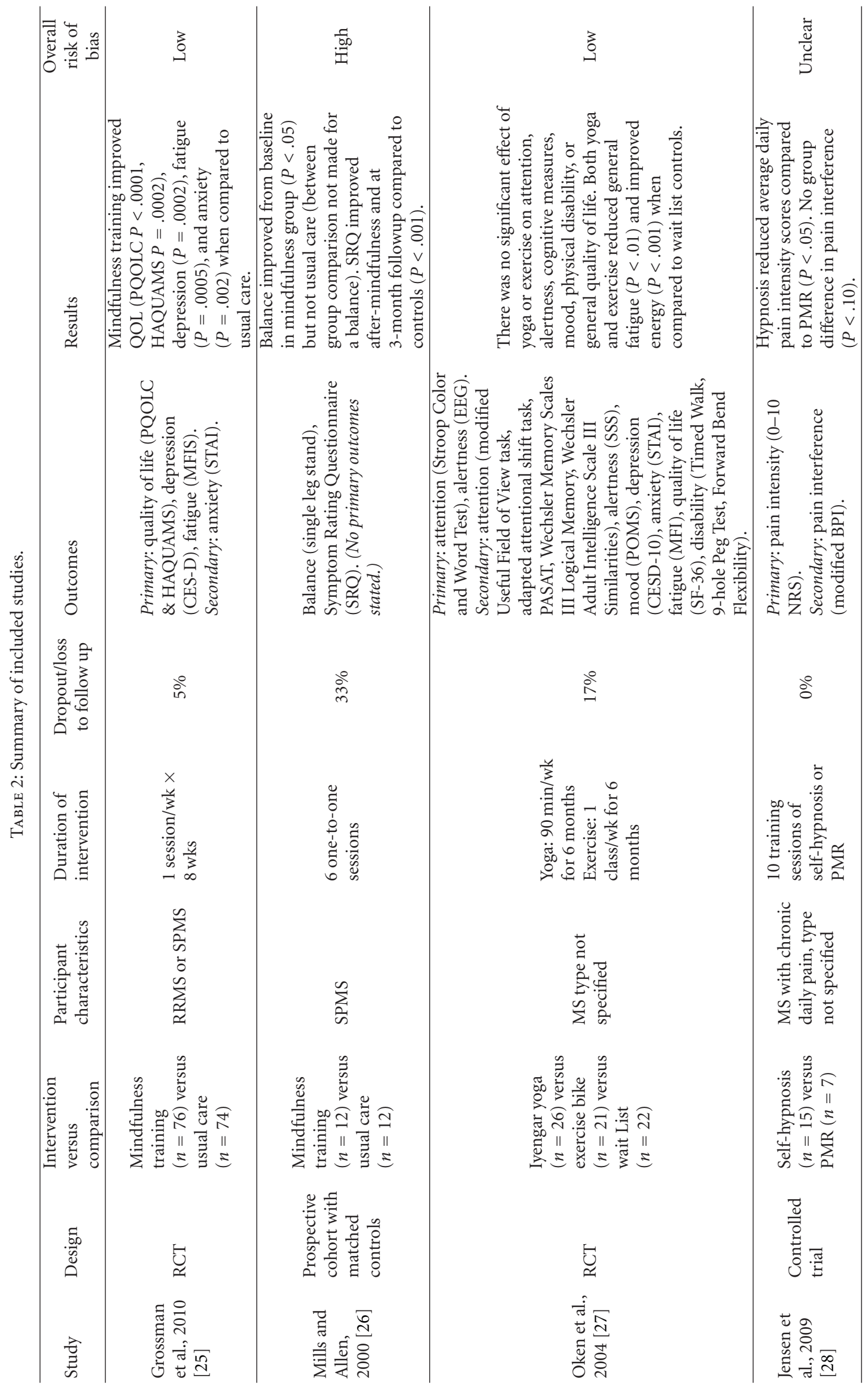




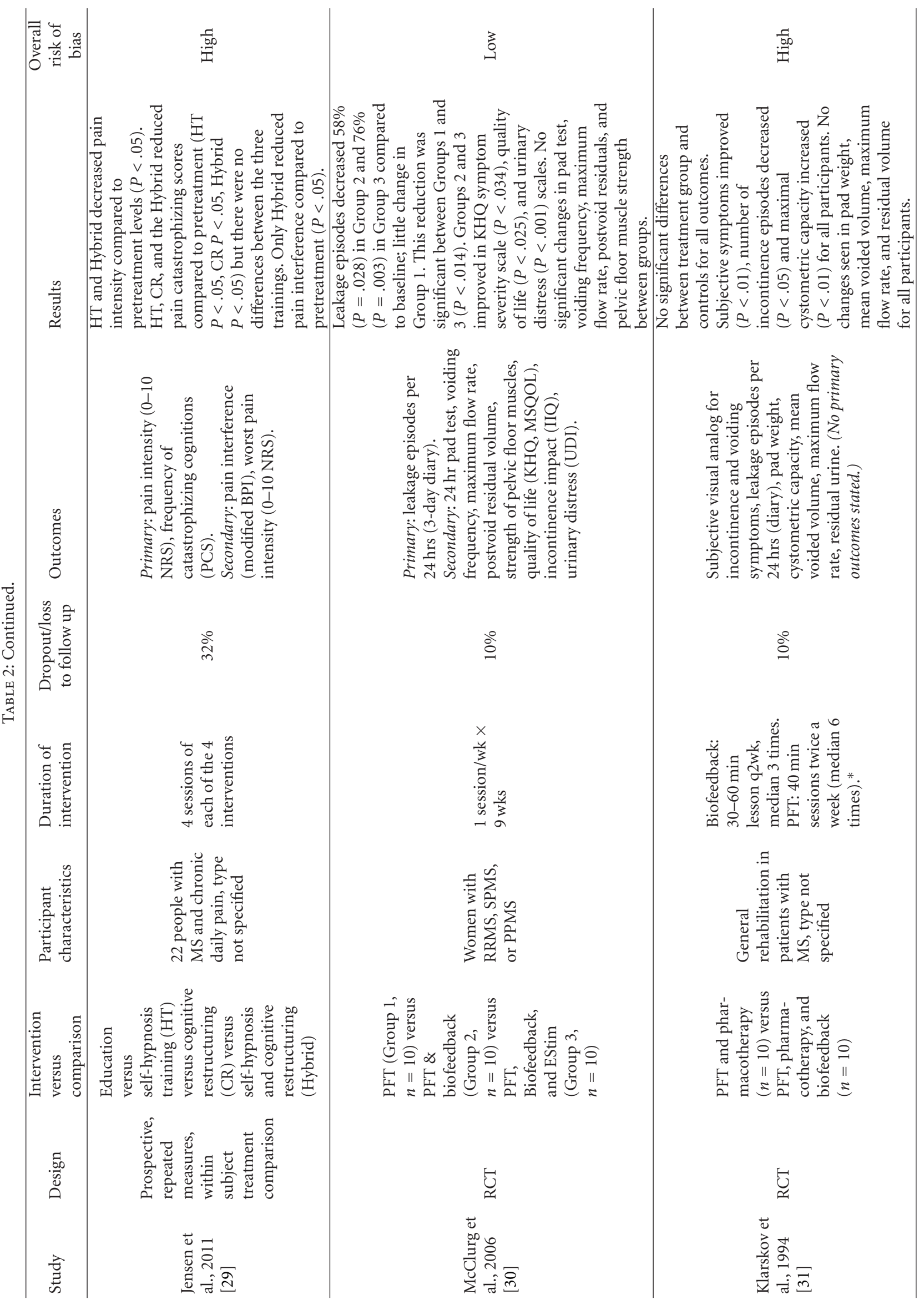




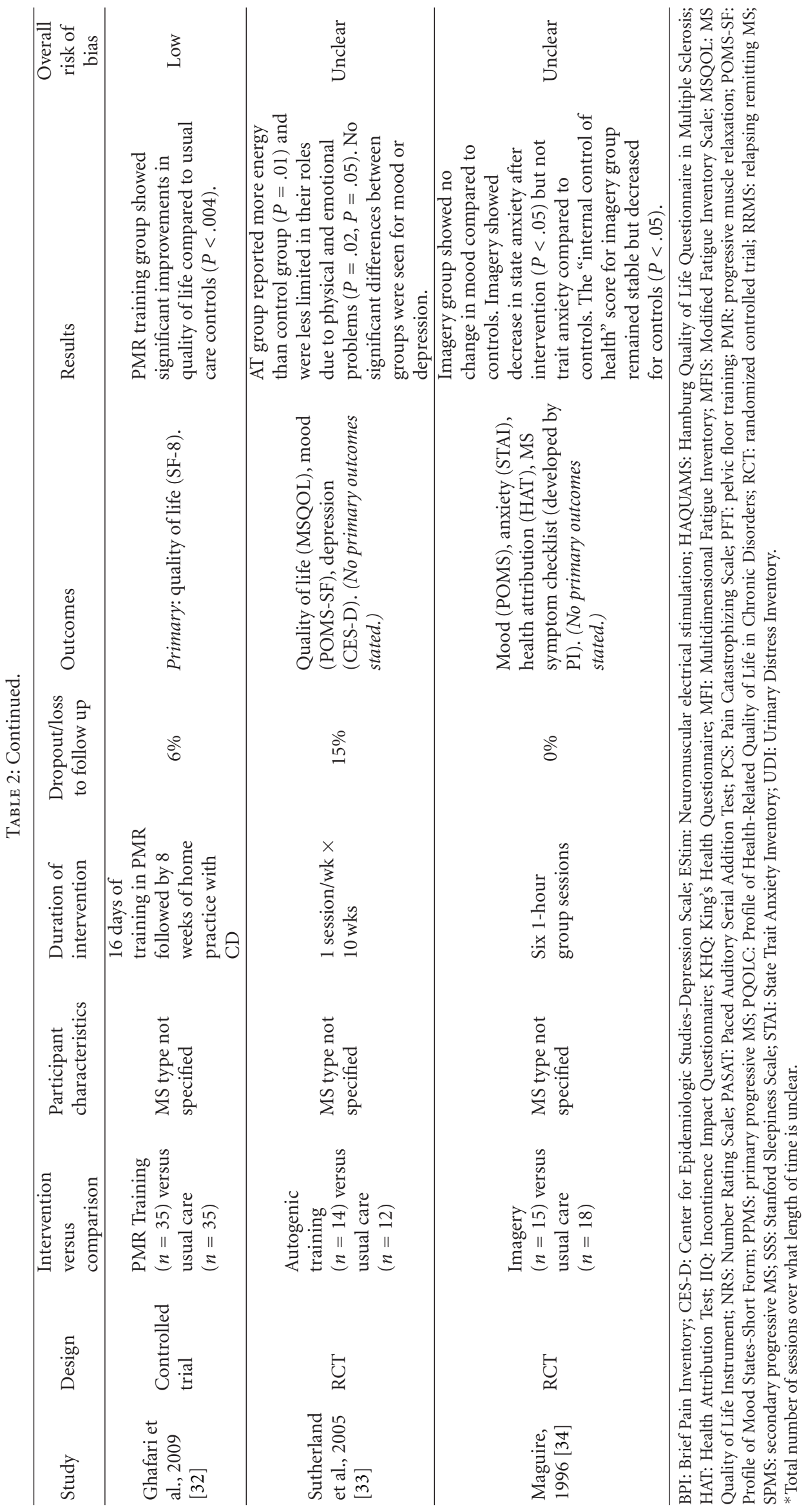




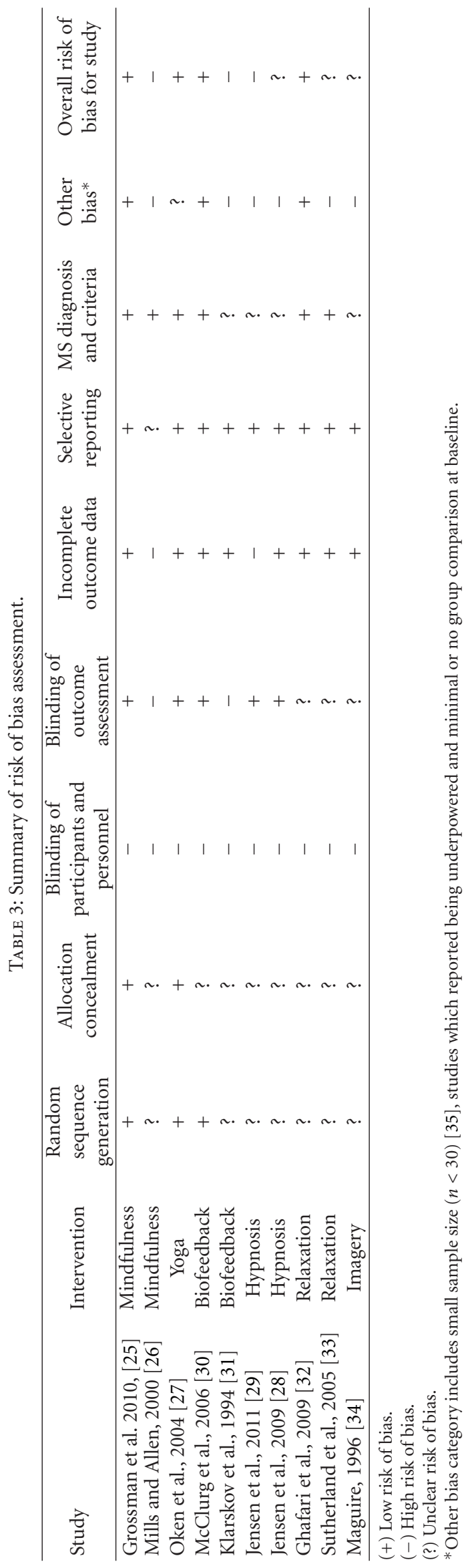


TABLE 4: Of 10 studies reviewed, four high quality studies were found helpful for symptoms of MS.

\begin{tabular}{lll}
\hline Study & Intervention & Helpful for \\
\hline $\begin{array}{l}\text { Ghafari et al., } \\
2009[32]\end{array}$ & Relaxation & Quality of Life \\
\hline $\begin{array}{l}\text { Grossman et al., } \\
2010 \text { [25] }\end{array}$ & $\begin{array}{l}\text { Mindfulness-based } \\
\text { stress reduction }\end{array}$ & $\begin{array}{l}\text { Depression } \\
\text { Anxiety } \\
\text { Fatigue } \\
\text { Quality of Life }\end{array}$ \\
\hline $\begin{array}{l}\text { Oken et al., } \\
2004 \text { [27] }\end{array}$ & Yoga & Fatigue \\
\hline $\begin{array}{l}\text { McClurg et al., } \\
2006 \text { [30] }\end{array}$ & Biofeedback & Bladder incontinence \\
\hline
\end{tabular}

consider the risk of bias of sufficient magnitude to have a notable impact on the results or conclusions of the trial. Several of the studies failed to provide enough detail for adequate assessment. Details regarding random sequence generation and allocation concealment were particularly poorly reported. Three studies did not provide sufficient details about baseline characteristics of comparison groups $[28,29,31]$. As it is impossible to blind participants to interventions that require their active involvement, all 10 studies received High risk of bias scores for this category. Sample sizes were generally small and seven of the studies had $\leq 33$ subjects. Only three studies provided intention to treat analysis $[25,27,30]$. Overall, we found four of the 10 studies to be methodologically sound [25, 27, 30, 32], three to have an Unclear risk of bias $[28,33,34]$, and three to have high overall risk of bias (Table 3 ) $[26,29,31]$.

\section{Discussion}

The objective of this paper was to assess the published evidence for using mind-body techniques for symptom management of multiple sclerosis. Four high quality studies showed that mindfulness, yoga, biofeedback, and relaxation had a positive effect on depression, anxiety, fatigue, quality of life, and bladder incontinence (Table 4) and no effect on disability, executive function, or other cognitive measures. The remaining studies demonstrated benefit for balance and daily pain intensity, but had no effect on executive function, mood, or disability - although many methodological inadequacies were identified. This paper demonstrates that studies of mind-body techniques for treating MS symptoms are feasible, and that more stringently designed, well-executed research is needed in this population to determine efficacy.

4.1. Implications for Research. More rigorous research is needed utilizing mind-body techniques for MS. Of the few studies identified in this paper, most were not of high quality, often due to small numbers and a lack of assessor blinding. In trials where it is impossible to blind participants to the study intervention, it is imperative to ensure that those assessing outcomes have no knowledge of the subjects' group assignment. Reporting on the blinding methodology for outcome assessors including maintenance of blinding throughout the study will improve the quality of future trials.

Placebo effects due to expectation are important to assess in trials where subjects are not blinded. Because subjects know which intervention group they will be participating in, it is possible that anticipation of improvement may influence outcomes. Only one study in this paper evaluated expectation effects. Jensen et al., 2009 found that expectancy was associated with a positive treatment outcome in response to self-hypnosis training for chronic pain. It is important to measure such relationships so that expectancy effects are distinguished from true intervention effects, and the impact of expectancy on outcome can be determined. Furthermore, such research may help identify ways to enhance expectancy in the clinical setting. In clinical practice when patients are given a known therapy, the effectiveness of the therapy is a combination of nonspecific and biologically active effects. Understanding the nonspecific effects that contribute to beneficial findings in trials (e.g., expectation, self-efficacy, motivation for improvement, locus of control, patient/provider relations, and attitudes toward one's disease and healing) will further our knowledge of mind-body therapies and enhance the generalizability of research trials to clinical practice.

The selection of appropriate comparison groups is critical when designing nonblinded intervention trials using mind-body techniques. Six studies in this paper used a nonintervention control group (either usual care or waitlist) [25-27, 32-34]. A limitation of this design is that it does not control for nonspecific factors involved in the treatment group like social effects of group work and attention from instructors [40]. A well-chosen active comparison group can control both treatment-specific and nonspecific effects of the study intervention, although this kind of comparison has disadvantages as well. A drawback to active controls is that they can exert their own therapeutic benefit beyond nonspecific social effects, thus making comparisons between groups difficult to interpret [41]. For example, Oken et al. compared yoga with an active control (exercise) to evaluate yoga-specific effects and control time and attention of the intervention and found few differences between groups. Exercise is known to have a therapeutic benefit in MS [42] and this could have been problematic had the authors not also included a nonintervention (waitlist) group for comparison. Three-arm trials that include both active and nonintervention controls are favorable if they can be afforded. Challenges regarding control group selection for mind-body medicine are not new and have been described by others $[41,43,44]$.

Level of disability should be considered during the design of a study. One example is highlighted by the Klarskov study [31]. The biofeedback protocol in this trial was specifically designed to enhance the awareness of muscles in the pelvic floor, yet 11 of the 20 subjects could not voluntarily contract pelvic floor muscles as measured by EMG, and 18 of the 20 subjects were unable to contract muscles voluntarily as measured by digital exam. The goal of the intervention was to use EMG feedback and yet a majority of the participants were unable to produce an EMG signal. Thus, the high 
baseline disability scores may have accounted for the absence of biofeedback effect between groups. Inclusion criteria for a baseline disability that allowed for an appropriate EMG feedback would have strengthened the study design and helped determine a therapeutic benefit.

While it may appear self-evident that mind-body therapies have a favorable risk-benefit ratio, only one study specifically discussed safety in terms of adverse events [27]. Four papers reported why subjects dropped out of the study, but furnished no other safety information $[25,26,30$, 33]. Based on limited data, no intervention-related safety issues were identified in this paper. Even though mindbody interventions are typically gentle and serious adverse events, unlikely, future trials should collect data necessary to calculate appropriate risk assessments.

In order to advance mind-body research for MS, future studies must attempt to adequately power their trials for an appropriate sample size. Only three studies reported power analyses a priori and as a result had adequate sample sizes $[25,27,32]$. The remaining trials did not benefit from power analyses and thus risked type II error, or false negative results.

4.2. Implications for Practice. Mind-body therapies are valuable because they can improve symptoms that affect quality of life. For example, fatigue is one of the most common and troubling symptoms in MS. MS-related fatigue is associated with decreased quality of life and depression and is described as the worst symptom of MS by $69 \%$ of patients [45]. It is estimated that 75-95\% of MS patients suffer from fatigue $[45,46]$ and pharmaceutical treatment provides limited relief. Our paper found that both yoga and mindfulness training improved MS fatigue, offering support with fewer side effects than conventional treatment. Therapies that can improve quality of life and the patient's ongoing experience of MS are essential to the successful management of this disease.

While there are not enough research studies to provide strong evidence for the use of mind-body therapies in MS, there is no evidence stating that they are harmful either. Patients who express interest in participating in such therapies do not necessarily need to be dissuaded because of harmful effects. However, clinicians should be mindful of a few key points when discussing mind-body medicine with MS patients.

(i) Hypnosis can allow distressing, repressed psychological content to come into consciousness and may not be appropriate for some patients with psychiatric disorders [47].

(ii) Guided or motor imagery requires that individuals imagine themselves performing a task, including the sensations that may accompany that activity and may not be suitable for patients with substantial cognitive impairment [48].

(iii) Yoga may not be appropriate for people with musculoskeletal conditions unless postures can be modified for individual circumstances. Props, such as cushions, blocks, and straps, can aid patients in holding poses, requiring less muscular effort. Bikram Yoga is practiced in a heated room (typically $105^{\circ}$ ) and risks exacerbating MS symptoms [49]. Working with a teacher who has experience adapting yoga practice for those with disabilities is advised.

(iv) Practitioner training and licensing may be an issue for some modalities. Health care providers can use biofeedback devices within the scope of practice defined by their license. Voluntary certification is available [50] for those who do not hold a health care license, but is not currently required by most states. Thus, training and experience may vary greatly from one therapist to the next and should be investigated. Hypnosis is typically practiced as an adjunct to psychotherapy by mental health care providers; however, most states do not require a license to practice and a hypnotherapist's credentials should be investigated. Patients should be advised to seek out appropriately trained providers for the modality they are interested in.

4.3. Limitations of This Paper. The current study had limitations that should be considered in interpreting the results. A single author performed database searches, conducted initial reviews, and extracted data; thus the initial study selection and data extraction may have been subject to bias and error. To address this, a librarian formulated a second search strategy that failed to retrieve any new articles (see the Appendix). That both search strategies retrieved identical results suggests no bias at the initial searching level. Only English language trials were included. Including foreign language search results may have contributed additional studies. Other studies may have been inadvertently missed because a manual search of references and reviews was not done. Authors were also not contacted for missing information. Regardless of these limitations, this is the first paper to assess the published research for mind-body medicine in MS. This is a starting point upon which one can build further assessment of the efficacy and feasibility of mind-body therapies for MS.

\section{Conclusion}

We found evidence to suggest that mind-body therapies are effective for treating common MS symptoms, including fatigue, anxiety, depression, incontinence, and quality of life. Mind-body modalities appear safe, can be prescribed as an adjunct to conventional care, and may be especially helpful when psychosocial stress is a factor or non-pharmacological options are desired (e.g., polypharmacy, pregnancy, and patient preference). More rigorously designed trials of mindbody interventions applied specifically to MS are needed in order to determine their efficacy and optimal selection for specific MS symptoms. Such research will enhance our understanding of the clinical effects of mind-body medicine as well as increase awareness and availability to clinicians and patients alike. 


\section{Appendix}

Two search strategies were applied to each electronic database: MEDLINE, PsycINFO, and Cochrane Clinical Trials Register.

(1) Hannon search strategy: (exp Mind-Body Therapies OR exp Mind-Body Relations, Metaphysical) AND (exp therapeutics OR th.fs.) AND exp multiple sclerosis OR multiple sclerosis, chronic progressive OR multiple sclerosis, relapsing-remitting).

(2) Senders search strategy: (exp multiple sclerosis OR multiple sclerosis, chronic progressive OR multiple sclerosis, relapsing-remitting) AND (qi gong.mp. OR exp Meditation OR mindfulness.mp. OR exp Relaxation OR exp Muscle Relaxation OR exp Relaxation Therapy OR exp Breathing Exercises OR breathing technique.mp. OR breathwork.mp. OR breathwork.mp. OR exp Hypnosis OR exp "Imagery (Psychotherapy)" OR exp Yoga OR exp Biofeedback, Psychology OR exp Tai Ji OR tai chi.mp.).

\section{Conflict of Interests}

The authors have no conflict of interests.

\section{Acknowledgments}

The authors thank Marian McDonagh for valuable direction and Todd Hannon for help with the search strategies. This work was supported by the Oregon Clinical and Translational Research Institute Grant number UL1 RR024140 from the National Center for Research Resources and the National Center for Advancing Translational Sciences of the National Institutes of Health, and the National Center for Complementary and Alternative Medicine of the National Institutes of Health Grant numbers AT002688, K01AT004951. The content of this paper is solely the responsibility of the authors and does not necessarily represent the official views of the National Institutes of Health.

\section{References}

[1] J. M. Buelow, "A correlational study of disabilities, stressors and coping methods in victims of multiple sclerosis," The Journal of Neuroscience Nursing, vol. 23, no. 4, pp. 247-252, 1991.

[2] M. A. Counte, L. A. Bieliauskas, and M. Pavlou, "Stress and personal attitudes in chronic illness," Archives of Physical Medicine and Rehabilitation, vol. 64, no. 6, pp. 272-275, 1983.

[3] H. M. Chalk, "Mind over matter: cognitive-behavioral determinants of emotional distress in multiple sclerosis patients," Psychology, Health and Medicine, vol. 12, no. 5, pp. 556-566, 2007.

[4] M. Korostil and A. Feinstein, "Anxiety disorders and their clinical correlates in multiple sclerosis patients," Multiple Sclerosis, vol. 13, no. 1, pp. 67-72, 2007.

[5] S. L. Minden, J. Orav, and P. Reich, "Depression in multiple sclerosis," General Hospital Psychiatry, vol. 9, no. 6, pp. 426434, 1987.
[6] D. S. P. Schubert and R. H. Foliart, "Increased depression in multiple sclerosis patients: a meta-analysis," Psychosomatics, vol. 34, no. 2, pp. 124-130, 1993.

[7] S. B. Patten, C. A. Beck, J. V. A. Williams, C. Barbui, and L. M. Metz, "Major depression in multiple sclerosis: a populationbased perspective," Neurology, vol. 61, no. 11, pp. 1524-1527, 2003.

[8] H. L. Zwibel and J. Smrtka, "Improving quality of life in multiple sclerosis: an unmet need," American Journal of Managed Care, vol. 17, supplement 5, pp. S139-S145, 2011.

[9] A. Riñon, M. Buch, D. Holley, and E. Verdun, "The MS Choices Survey: findings of a study assessing physician and patient perspectives on living with and managing multiple sclerosis," Patient Prefer Adherence, vol. 5, pp. 629-643, 2011.

[10] P. M. Barnes, B. Bloom, and R. L. Nahin, "Complementary and alternative medicine use among adults and children: United States, 2007," National Health Statistics Reports, no. 12, pp. 123, 2009.

[11] V. Yadav, L. Shinto, C. Morris, A. Senders, S. Baldauf-Wagner, and D. Bourdette, "Use and self-reported benefit of complementary and alternative medicine among multiple sclerosis patients," International Journal of Multiple Sclerosis Care, vol. 8, pp. 5-10, 2006.

[12] "What is complementary and alternative medicine?" 2012, http://nccam.nih.gov/health/whatiscam.

[13] A. G. Taylor, L. E. Goehler, D. I. Galper, K. E. Innes, and C. Bourguignon, "Top-down and bottom-up mechanisms in mind-body medicine: development of an integrative framework for psychophysiological research," Explore, vol. 6, no. 1, pp. 29-41, 2010.

[14] J. A. Astin, S. L. Shapiro, D. M. Eisenberg, and K. L. Forys, "Mind-body medicine: state of the science, implications for practice," Journal of the American Board of Family Practice, vol. 16, no. 2, pp. 131-147, 2003.

[15] H. Wahbeh, S. M. Elsas, and B. S. Oken, "Mind-body interventions: applications in neurology," Neurology, vol. 70, no. 24, pp. 2321-2328, 2008.

[16] T. Esch, G. L. Fricchione, and G. B. Stefano, "The therapeutic use of the relaxation response in stress-related diseases," Medical Science Monitor, vol. 9, no. 2, pp. RA23-RA34, 2003.

[17] H. Benson, J. F. Beary, and M. P. Carol, "The relaxation response," Psychiatry, vol. 37, no. 1, pp. 37-46, 1974.

[18] R. P. Brown and P. L. Gerbarg, "Sudarshan Kriya yogic breathing in the treatment of stress, anxiety, and depressionpart I-neurophysiologic model," Journal of Alternative and Complementary Medicine, vol. 11, no. 1, pp. 189-201, 2005.

[19] S. Braun, M. Kleynen, J. Schols, T. Schack, A. Beurskens, and D. Wade, "Using mental practice in stroke rehabilitation: a framework," Clinical Rehabilitation, vol. 22, no. 7, pp. 579$591,2008$.

[20] J. Decety and M. Jeannerod, "Mentally simulated movements in virtual reality: does Fitt's law hold in motor imagery?" Behavioural Brain Research, vol. 72, no. 1-2, pp. 127-134, 1996.

[21] B. Wuyam, S. H. Moosavi, J. Decety, L. Adams, R. W. Lansing, and A. Guz, "Imagination of dynamic exercise produced ventilatory responses which were more apparent in competitive sportsmen," Journal of Physiology, vol. 482, no. 3, pp. 713-724, 1995.

[22] S. Thobois, P. F. Dominey, J. Decety et al., "Motor imagery in normal subjects and in asymmetrical Parkinson's disease: a PET study," Neurology, vol. 55, no. 7, pp. 996-1002, 2000.

[23] M. Bonnet, J. Decety, M. Jeannerod, and J. Requin, "Mental simulation of an action modulates the excitability of spinal 
reflex pathways in man," Cognitive Brain Research, vol. 5, no. 3, pp. 221-228, 1997.

[24] L. Fadiga, G. Buccino, L. Craighero, L. Fogassi, V. Gallese, and G. Pavesi, "Corticospinal excitability is specifically modulated by motor imagery: a magnetic stimulation study," Neuropsychologia, vol. 37, no. 2, pp. 147-158, 1998.

[25] P. Grossman, L. Kappos, H. Gensicke et al., "MS quality of life, depression, and fatigue improve after mindfulness training: a randomized trial," Neurology, vol. 75, no. 13, pp. 1141-1149, 2010.

[26] N. Mills and J. Allen, "Mindfulness of movement as a coping strategy in multiple sclerosis. A pilot study," General Hospital Psychiatry, vol. 22, no. 6, pp. 425-431, 2000.

[27] B. S. Oken, S. Kishiyama, D. Zajdel et al., "Randomized controlled trial of yoga and exercise in multiple sclerosis," Neurology, vol. 62, no. 11, pp. 2058-2064, 2004.

[28] M. P. Jensen, J. Barber, J. M. Romano et al., "A comparison of self-hypnosis versus progressive muscle relaxation in patients with multiple sclerosis and chronic pain," International Journal of Clinical and Experimental Hypnosis, vol. 57, no. 2, pp. 198 221, 2009.

[29] M. P. Jensen, D. M. Ehde, K. J. Gertz et al., "Effects of selfhypnosis training and cognitive restructuring on daily pain intensity and catastrophizing in individuals with multiple sclerosis and chronic pain," International Journal of Clinical and Experimental Hypnosis, vol. 59, no. 1, pp. 45-63, 2011.

[30] D. McClurg, R. G. Ashe, K. Marshall, and A. S. Lowe-Strong, "Comparison of pelvic floor muscle training, electromyography biofeedback, and neuromuscular electrical stimulation for bladder dysfunction in people with multiple sclerosis: a randomized pilot study," Neurourology and Urodynamics, vol. 25, no. 4, pp. 337-348, 2006.

[31] P. Klarskov, E. Heely, I. Nyholdt, K. Rottensten, and A. Nordenbo, "Biofeedback treatment of bladder dysfunction in multiple sclerosis. A randomized trial," Scandinavian Journal of Urology and Nephrology, no. 157, pp. 61-65, 1994.

[32] S. Ghafari, F. Ahmadi, M. Nabavi, K. Anoshirvan, R. Memarian, and M. Rafatbakhsh, "Effectiveness of applying progressive muscle relaxation technique on quality of life of patients with multiple sclerosis," Journal of Clinical Nursing, vol. 18, no. 15, pp. 2171-2179, 2009.

[33] G. Sutherland, M. B. Andersen, and T. Morris, "Relaxation and health-related quality of life in multiple sclerosis: the example of autogenic training," Journal of Behavioral Medicine, vol. 28, no. 3, pp. 249-256, 2005.

[34] B. L. Maguire, "The effects of imagery on attitudes and moods in multiple sclerosis patients," Alternative Therapies in Health and Medicine, vol. 2, no. 5, pp. 75-79, 1996.

[35] R. A. Moore, D. Gavaghan, M. R. Tramèr, S. L. Collins, and H. J. McQuay, "Size is everything-large amounts of information are needed to overcome random effects in estimating direction and magnitude of treatment effects," Pain, vol. 78, no. 3, pp. 209-216, 1998.

[36] J. P. T. Higgins, D. G. Altman, P. C. Gøtzsche, P. Jüni, D. Moher, and A. D. Oxman, "The Cochrane Collaboration's tool for assessing risk of bias in randomised trials," British Medical Journal, vol. 343, Article ID d5928, 2011.

[37] J. L. Broek, E. A. Akl, P. Alonso-Coello et al., "Grading quality of evidence and strength of recommendations in clinical practice guidelines - part 1 of 3 . An overview of the GRADE approach and grading quality of evidence about interventions," Allergy, vol. 64, no. 5, pp. 669-677, 2009.

[38] W. I. McDonald, A. Compston, G. Edan et al., "Recommended diagnostic criteria for multiple sclerosis: guidelines from the
International panel on the diagnosis of multiple sclerosis," Annals of Neurology, vol. 50, no. 1, pp. 121-127, 2001.

[39] J. F. Kurtzke, "Rating neurologic impairment in multiple sclerosis: an expanded disability status scale (EDSS)," Neurology, vol. 33, no. 11, pp. 1444-1452, 1983.

[40] Z. Di Blasi, E. Harkness, E. Ernst, A. Georgiou, and J. Kleijnen, "Influence of context effects on health outcomes: a systematic review," Lancet, vol. 357, no. 9258, pp. 757-762, 2001.

[41] W. E. Whitehead, "Control groups appropriate for behavioral interventions," Gastroenterology, vol. 126, no. 1, supplement1, pp. S159-S163, 2004.

[42] U. Dalgas, E. Stenager, and T. Ingemann-Hansen, "Multiple sclerosis and physical exercise: recommendations for the application of resistance-, endurance- and combined training," Multiple Sclerosis, vol. 14, no. 1, pp. 35-53, 2008.

[43] M. B. Ospina, K. Bond, M. Karkhaneh et al., "Clinical trials of meditation practices in health care: characteristics and quality," Journal of Alternative and Complementary Medicine, vol. 14, no. 10, pp. 1199-1213, 2008.

[44] P. Mansky, T. Sannes, D. Wallerstedt et al., "Tai chi chuan: mind-body practice or exercise intervention? Studying the benefit for cancer survivors," Integrative Cancer Therapies, vol. 5, no. 3, pp. 192-201, 2006.

[45] H. L. Zwibel, "Contribution of impaired mobility and general symptoms to the burden of multiple sclerosis," Advances in Therapy, vol. 26, no. 12, pp. 1043-1057, 2009.

[46] T. Ziemssen, "Multiple sclerosis beyond EDSS: depression and fatigue," Journal of the Neurological Sciences, vol. 277, no. 1, supplement, pp. S37-S41, 2009.

[47] K. Barrows, Complementary \& Alternative Medicine. Current Medical Diagnosis \& Treatment, McGraw-Hill, 2012.

[48] C. L. Hovington and B. Brouwer, "Guided motor imagery in healthy adults and stroke: does strategy matter?" Neurorehabilitation and Neural Repair, vol. 24, no. 9, pp. 851-857, 2010.

[49] T. C. Guthrie and D. A. Nelson, "Influence of temperature changes on multiple sclerosis: critical review of mechanisms and research potential," Journal of the Neurological Sciences, vol. 129, no. 1, pp. 1-8, 1995.

[50] "Biofeedback Certification International Alliance," 2012, http://www.bcia.org/i4a/pages/index.cfm?pageid=1. 


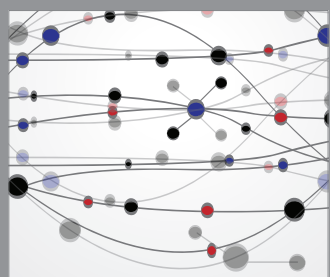

The Scientific World Journal
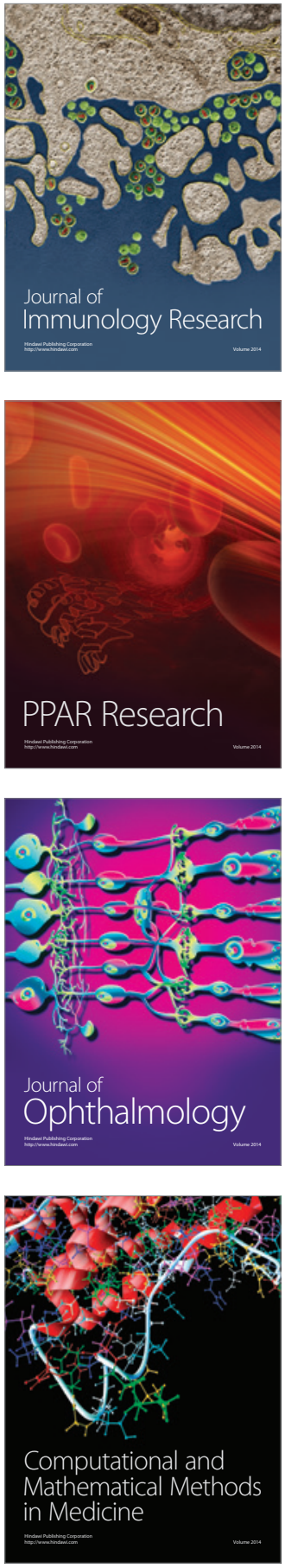

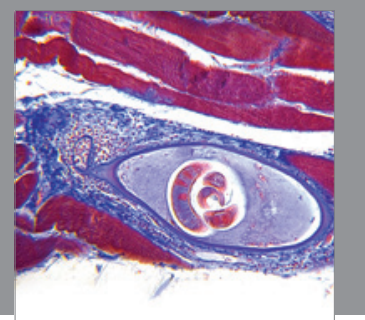

Gastroenterology

Research and Practice
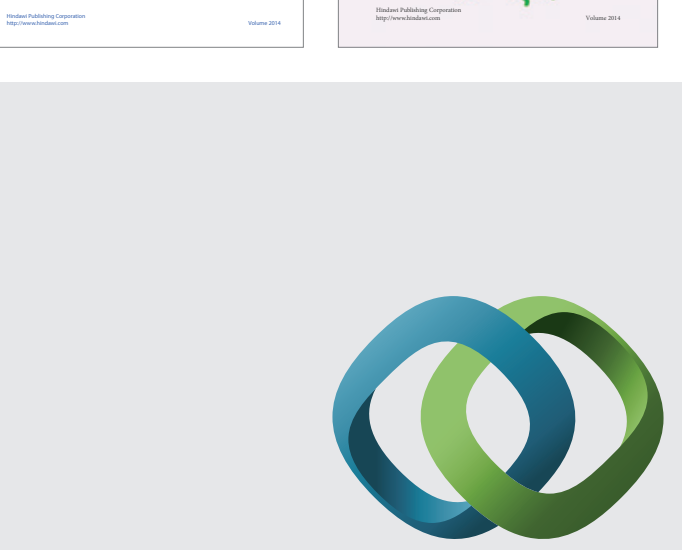

\section{Hindawi}

Submit your manuscripts at

http://www.hindawi.com
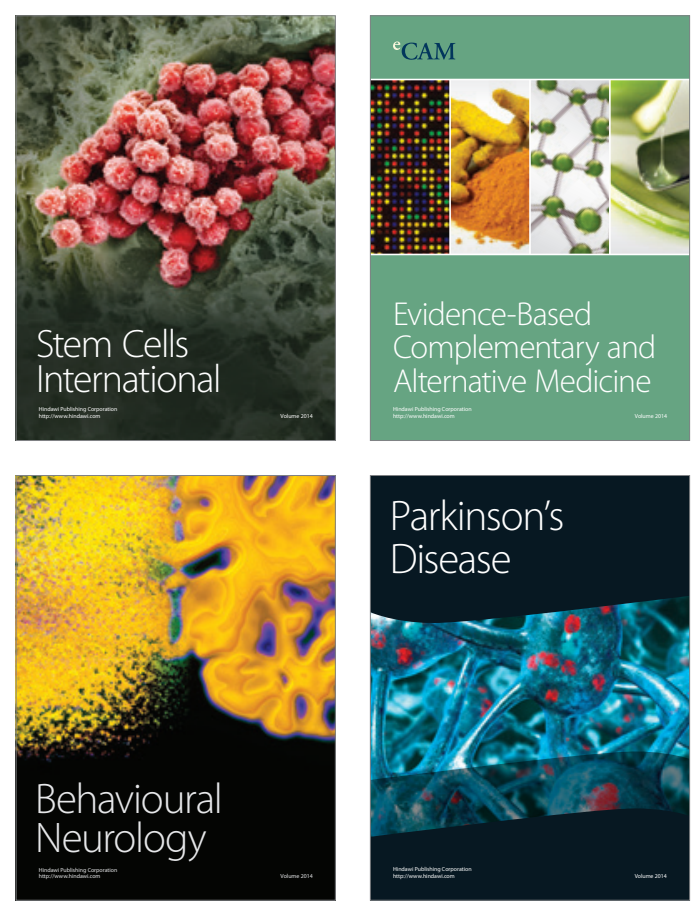

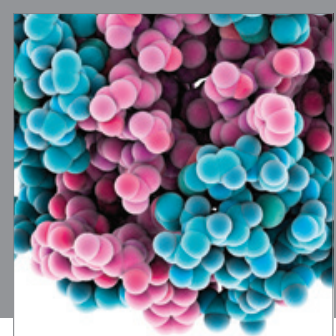

Journal of
Diabetes Research

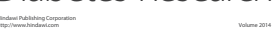

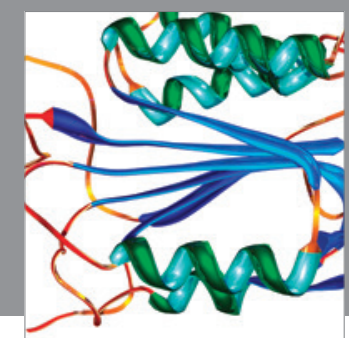

Disease Markers
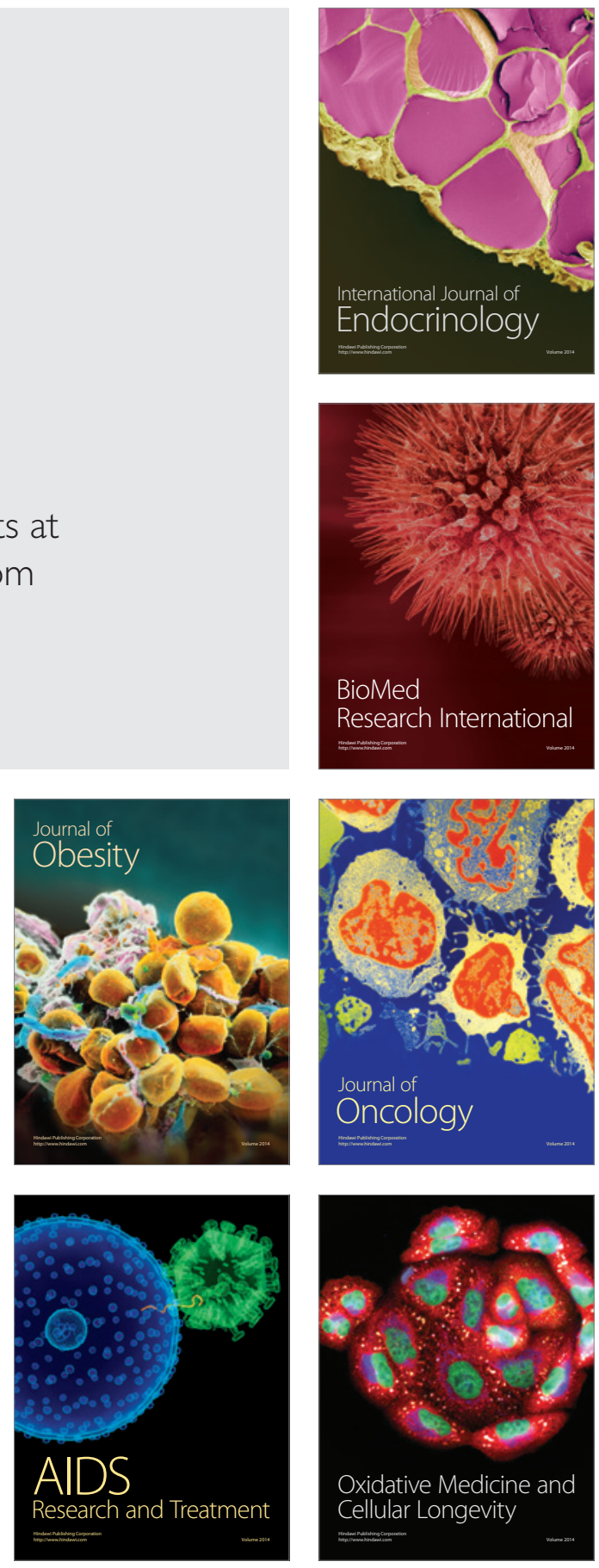